\title{
La radio cubana ante la COVID-19. Un estudio de caso
}

\section{The cuban radio against the COVID-19. A case study}

\author{
Beatriz Elena Fonseca Muñoza \\ a Departamento de Comunicación Social, Facultad de Comunicación, Universidad de La Habana, Cuba
}

\section{Resumen}

La presencia del nuevo coronavirus desde diciembre de 2019 ha transformado las dinámicas de vida a nivel mundial. En su enfrentamiento multisectorial, también intervienen los procesos de comunicación que se desarrollan en todos los medios, entre ellos la radio. Caracterizar cómo se hace la comunicación sobre la COVID-19 en la programación musical de la emisora Radio Progreso, es el objetivo del presente trabajo. Se aplica un análisis de contenido a dos programas musicales de radio para mostrar el modo de emplear los recursos expresivos del medio y el formato para hacerlo. Se constata que tanto las cuñas de radio como las intervenciones de locutores/ as, especialistas e invitados coincidieron en la orientación y sensibilización de los ciudadanos/as para asumir una actitud responsable en aras de vencer la crisis sanitaria. Estas acciones comunicativas consolidan el binomio comunicación y salud en una variante lúdico/educativa, para poder vencer la actual pandemia.

Palabras clave: Radio cubana; COVID-19; comunicación; salud.

\begin{abstract}
The presence of the new coronavirus since December 2019 has transformed life dynamics worldwide. In their multi-sectoral confrontation, the communication processes that take place in all media, including radio, also intervene. To characterize how communication about COVID-19 is done in the musical programming of the Radio Progreso station is the objective of this work. A content analysis is applied to two radio music programs to show how to use the expressive resources of the medium and the format to do so. It is verified that both the radio spots and the interventions of broadcasters, specialists and guests agreed on the orientation and sensitization of citizens to assume a responsible attitude in order to overcome the health crisis. These communication actions consolidate the communication and health binomial into a recreational / educational variant, in order to overcome the current pandemic.
\end{abstract}

Keywords: Cuban radio; COVID-19; communication; health. 


\section{Introducción}

a presencia del nuevo coronavirus SARS-CoV-2 ha transformado la vida cotidiana de gran parte de los países del planeta. Formas de vida, hábitos, horarios, entre otros, ha sido necesario cambiarlos para enfrentar un enemigo que no se ve. Los estragos del COVID-19 son incalculables por ser desconocido como patología, por su nivel de contagio y lo lejano del momento para tener una vacuna contra la enfermedad, pues la ciencia requiere de un tiempo determinado para obtenerla, como han explicado algunos especialistas.

Las circunstancias impuestas por este coronavirus exigen un trabajo multisectorial, en el caso cubano, encabezado por el Ministerio de Salud Pública (MINSAP). Como parte de este enfoque, se encuentra la labor que desarrolla el Instituto Cubano de Radio y Televisión (ICRT), sistema de comunicación de carácter público, en la comunicación permanente sobre el tema a la población del país. Para lo cual emplea su sistema nacional, provincial y municipal; subsistemas de la televisión y la radio, así como las más diversas formas de producción comunicativa y ejercicios profesionales.

Desde que en el mes de marzo de 2020 se dieran a conocer los primeros casos de COVID-19 en Cuba, la radio desarrolló su comunicación dirigida a orientar, educar, recrear, informar y elevar la cultura de la ciudadanía, a través de sus 100 emisoras (una internacional, seis nacionales, 19 provinciales y 74 municipales) y 68 sitios webs. De las seis nacionales, Radio Progreso, la "emisora de la familia cubana", constituye el centro de este artículo, cuyo objetivo general es caracterizar la comunicación que realiza sobre la COVID-19 en su programación musical.

Todo ello bajo el precepto de que la radio es eficaz para informar, transmitir conocimientos y promover inquietudes. Asimismo, permite la reflexión sobre valores y actitudes y la formación de una conciencia crítica (Kaplún, 2005, p. 86).

De "El Progreso Cubano" a "Onda de la Alegría"

Llamada "El Progreso Cubano", esta emisora inicia sus primeras emisiones el 15 de diciembre de 1929. En 1931 adopta el nombre de Radio Progreso, la "Onda de la Alegría". Antes del triunfo revolucionario contaba con anunciantes o firmas que patrocinaban los espacios más preferidos por la audiencia, por lo que la publicidad comercial era el soporte de su programación, que abarcaba diversos tipos de espacios: musicales, campesinos, infantiles y dramatizados, entre otros.

Desde la década del cincuenta del siglo XX, se reconoce como "la casa de la Orquesta Aragón", la cual interpreta el tema de presentación de la emisora hasta la actualidad. La letra es un poema de Jesús Orta Ruíz, el Indio Naborí, creado con este objetivo y con música de Rafael Lay, padre, Director de la Orquesta Aragón. Entre 1961 y 1963, en sus estudios grababan orquestas dirigidas por los maestros Rodrigo Prats, Adolfo Guzmán y Mario Romeu, con un variado repertorio de música popular, zarzuelas, entre otras.

\section{Entre publicidad, propaganda y la promoción}

Sin hacer un análisis exhaustivo sobre la diferencia entre publicidad y propaganda, pues no es el objetivo del trabajo y el espacio para ello es insuficiente, se considera importante reflexionar sobre algunas ideas dado el contexto social en el que se circunscribe el artículo.

En la sociedad cubana, antes del 1 de enero de 1959, la publicidad estuvo presente en las relaciones de producción capitalista dominantes y representaba sus intereses, por tanto, definían las políticas en la información. Así lo destaca Fidel en octubre de ese mismo año en su comparecencia ante la prensa, "El monopolio de la publicidad, el monopolio de la información estaba en manos de ese mismo grupo que tenía el monopolio de la riqueza" (Castro en Muñiz, 1990, p.23). Las técnicas publicitarias respondían a los rasgos de una sociedad signada por las relaciones comerciales con los Estados Unidos. En la década del 50 se desarrolló la televisión, la que en sus inicios emplea las técnicas radiales de hacer publicidad.

El triunfo de la Revolución Cubana pone a la actividad publicitaria al servicio de los objetivos de la nueva sociedad que surgía; pero, a decir de Muñiz (1999), los monopolios tradicionales publicitarios no asumieron estos retos y quedaron apartados de las exigencias del naciente modo de hacer. Finalmente, la publicidad comercial desaparece del escenario nacional hasta los años 90 que es retomada debido a los cambios sociales que trae aparejado el llamado Período Especial, con el derrumbe del campo socialista y la desaparición de la Unión Soviética, lo que influye en el desarrollo económico, político y social del país.

En 1985 se creó Radio Taíno, dirigida al turismo internacional, aunque en la actualidad alcanza a la audiencia cubana. Las nuevas condiciones económicas incorporan una visión diferente al fenómeno publicitario, dado el compromiso social de la radio con la sociedad (Fonseca y Saladrigas, 2016, p. 107). Esta emisora mantiene la publicidad; pero no es la esencia de su programación. Las demás emisoras desarrollan la comunicación propagandística y de bien público.

El término propaganda se relaciona, históricamente, con la manipulación que se hace a las audiencias y, 
por ello, no goza de buena reputación en la historia del desarrollo del pensamiento social, para Chomsky "Los medios de comunicación son a la democracia lo que la propaganda es a la dictadura" (citado por Ramonet, 2016, p. 128).

En la Radio Cubana, esta actividad se dirige desde la Dirección de Información y Propaganda y durante más de veinte años, hasta su desaparición física, fue estructurada y organizada por Juan Hernández Rodríguez, quien también la fundamentó teórica y metodológicamente, hasta donde su experiencia le permitió. Partiendo de la concepción de la Propaganda como Directa e Indirecta ${ }^{1}$ la primera la enmarca en sus formas de realización (Menciones, Promociones, Mensajes Cortos y Jingles).

La Mención tiene como objetivo fijar una idea en la conciencia de la audiencia. Llama la atención inmediata, a modificar una actitud, mantener o modificar una conducta. Debe hacer una síntesis de contenido, emplear la palabra viva y complementos que pueden ser utilizados o no, como la música, los efectos, el silencio. Su tiempo total no debe exceder los 30 segundos y, con excepción, 45 segundos.

La Promoción ofrece opciones de eventos, actos públicos, actividades, programaciones, entre otros, de carácter cultural, políticos y sociales, que pueden ser de interés conocer para su posterior selección y asistencia. En ella debe considerarse: 1) Que el contenido emplee apelaciones, motivaciones y gratificaciones que logre la audiencia; 2) Que la información responda a las preguntas: cuándo, dónde y cómo podré optar por la propuesta. No exceder los 45 segundos y por excepción, 1 minuto.

El Jingle tiene por objetivo, trasladar una idea sobre una temática, producto o servicio (en el caso de la publicidad) con música. La concepción musical debe ser original y no de elaboración simple. Su letra debe comunicar lo esencial de la idea a trasladar y la gratificación que le ofrece su aceptación.

Los Mensajes Cortos se caracterizan por dar un consejo, conocimiento, definición, sobre una temática. Si bien cada mensaje corto es independiente en cuanto a contenido en sí, responde a un seriado temático y requiere: 1) un pequeño corte musical, al inicio y final del mensaje, que se convierta en un logotipo musical de la temática en cuestión; 2) una o dos veces (si el mensaje es muy largo 0 su texto lo requiere) interpretarán la síntesis de contenido propuesto y 3) no se emplearán efectos, dramatizaciones $u$ otros recursos radiales que no sea la locución.

1 Documentos Metodológicos de la Dirección de Información y Propaganda de la Radio Cubana.
La Propaganda Indirecta, es efectiva para lograr introducir conocimientos en los oyentes, ideas que les sugieren adoptar, cambiar o modificar su forma de conducirse individual o socialmente. Esta propaganda se identifica en las diversas voces que intervienen en los programas: locutoras, locutores, periodistas, especialistas de otras áreas que intervienen (salud pública, educación, transporte, entre otros). La propaganda indirecta posibilita el tratamiento de los temas de un modo más amplio y, con ello, la interacción, en ocasiones, con los radioyentes, a partir del tipo de programa.

Esta clasificación de la propaganda organiza la producción comunicativa de radio, según la complejidad de las temáticas y la definición de los formatos más adecuados para su salida al aire, atendiendo al tema, perfil y objetivos de los programas y audiencias a las que se transmiten.

Al evaluarse estas "formas simbólicas" o "productos comunicativos", se analizan todos los recursos expresivos que se emplean en su producción: voz, música, efectos, sonidos y silencios. Cada uno de ellos estructurado según el formato y género de radio de que se trate.

Si bien en la academia el término publicidad de bien público cada día tiene mayor presencia, en el contexto de la radio cubana aún se reconoce el de propaganda en sentido político y de bien público. En el presente artículo se asume la conceptualización de Hernández Rodríguez ya identificada. Esta es más cercana al contexto que aquí se hace referencia y, por ello, se corresponde de forma más exacta.

Para caracterizar la comunicación sobre la COVID-19 que hiciera Radio Progreso, se aplicó el análisis de contenido, definido por Piñuel (1995, p.2) como:

Conjunto de procedimientos interpretativos de productos comunicativos (mensajes, textos o discursos) que proceden de procesos singulares de comunicación previamente registrados, y que, basados en técnicas de medida, a veces cuantitativas (estadísticas basadas en el recuento de unidades), a veces cualitativas (lógicas basadas en la combinación de categorías) tienen por objeto elaborar y procesar datos relevantes sobre las condiciones mismas en que se han producido aquellos textos, o sobre las condiciones que puedan darse para su empleo posterior (Piñuel, 1995, p.2) .

Siguiendo la clasificación de este mismo autor, se está ante un análisis de contenido descriptivo, que identifica y cataloga la realidad empírica estudiada y, al mismo tiempo, es del tipo vertical o intensivo, donde el significado se deriva de las relaciones, las oposiciones y el contexto, no así de medidas cuantitativas (Piñuel, 1995). 


\section{Metodología}

Para el análisis de contenido fueron seleccionados los programas Juventud 2000 y La Discoteca Popular, por ser representativos de esta programación en la emisora y por su extensión. Se les hizo un monitoreo que suman 38 horas y 10 minutos, desde el lunes 27 de abril al sábado 2 de mayo de 2020, para grabar las emisiones que serían objeto de análisis. Estos días responden al hecho de que el domingo La Discoteca Popular no aparece programada en parrilla.

El horario de transmisión de Juventud 2000 comprende de lunes a jueves y sábado de 8:00 a 12:00 horas, pues el viernes Primero de mayo se amplió una hora hasta las 13:00 horas. Mientras La Discoteca Popular lo hace de lunes a jueves y sábado de 15:00 a 17:40 horas y viernes 1 de mayo de 13:30 a 18:00 horas.

La comunicación sobre la COVID-19 en la programación musical de radio

Se prefirió la programación musical para el análisis, porque las características de esta forma de hacer radio es a veces un argumento de sus realizadores para no tratar determinados temas $^{2}$. El análisis de contenido exigió que se definieran las siguientes variables y categorías correspondientes, según los objetivos formulados (Tabla 1).

Las 38 horas y 10 minutos monitoreadas reflejan la transmisión de 28 realizaciones de Propaganda Directa, distribuidas entre 23 menciones sobre el tema de la COVID-19, 3 relacionadas con otras temáticas: pensar como país, la denuncia al bloqueo económico y financiero del gobierno de los Estados Unidos y la importancia del amor en el desarrollo de la vida cotidiana para un $11 \%$ y 2 promociones sobre la Discoteca y la emisora que representan el $7 \%$.

En relación con la temática de la COVID-19 el comportamiento es como sigue: 12 menciones para un $43 \%, 6$ Mensajes Cortos que representan el $21 \%$ y 5 jingles para un $18 \%$ (Figura 1).

De las 12 menciones, 5 se refieren a la prevención de la COVID-19; 3 a las medidas para reutilizar el nasobuco de tela y su uso adecuado; 1 sobre la necesidad de que los especialistas de salud mantengan las medidas de protección para reducir los riesgos de enfermar y de la propagación del coronavirus; 1 sobre la necesidad de quedarse en casa y hacer cosas para la que antes no se tenía; pero velando por el ahorro energético, fundamentalmente, en el horario pico cuando es mayor

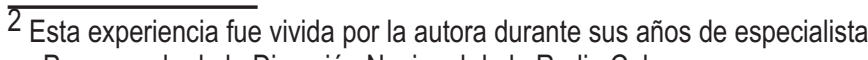
en Propaganda de la Dirección Nacional de la Radio Cubana. la demanda; 1 reflejando palabras de Fidel expresando la disposición de Cuba de ayudar a otros pueblos del mundo con médicos y no con bombas; y 1 sobre el primero de mayo relacionado con la lucha contra la COVID- 19.

Los 6 mensajes cortos se distribuyen en 2, donde psicólogas profesoras de la Universidad de La Habana, explican cómo enfrentar la situación de aislamiento y 4 donde artistas cubanos reconocidos sugieren qué hacer en el período de aislamiento y llamando a quedarse en casa.

De los 5 jingles, 1 es sobre las medidas de prevención, utilizando la música campesina; 2 muestran a cantantes exhortando a quedarse en casa; 1 sobre la necesidad de que los médicos se protejan; y 1 promociona a la Discoteca unido al llamado de quedarse en casa.

La propaganda indirecta en las voces de los locutores/ as mantuvo el llamado a una actitud responsable ante la pandemia, a informarse con las fuentes oficiales, cumplir las orientaciones de las autoridades sanitarias y del Estado y el Gobierno, a mantener las medidas higiénicas y, como algo fundamental, no salir de casa y hacerlo solo en caso necesario.

Estas ideas también se manifiestan en las entrevistas hechas, vía telefónica generalmente, a personalidades invitadas, que desde sus casas reiteran lo imprescindible de asumir esa actitud responsable.

En la emisión de Juventud 2000 del día 28 de abril, a la sección sobre El adulto mayor y la COVID-19, se invitó a un Doctor, gineco-obstetra, profesor auxiliar del Instituto Superior de Ciencias Médicas, Presidente de la Cátedra de sexología de la Facultad Comandante Manuel Fajardo, Máster en sexualidad, Máster en intervención comunitaria, especialista de PROSALUD ${ }^{3}$ (promoción de salud y prevención de enfermedades). Esta sección sale al aire todas las semanas.

Este especialista refiere que es una enfermedad nueva y menciona los resultados de algunos estudios sobre las complicaciones en personas adultas mayores, debido al estado de su sistema inmunológico. Fundamenta la necesidad del cuidado de estas personas, la importancia de hacer ejercicios con orientación de su médico de familia, si antes no los había hecho. Insiste en que deben informarse por las vías oficiales, buscar la información necesaria, no saturarse al respecto, no auto medicarse, velar por su nutrición, no encamarse, por supuesto, todo esto con la ayuda de familiares y cuidadores.

\footnotetext{
3 Unidad de Promoción de Salud y Prevención de Enfermedades creada el 30 de enero de 2014 por Resolución no. 33 del MINSAP y cuya misión es "Desarrollar, implementar y evaluar estrategias de promoción de salud y prevención de enfermedades, con énfasis en los grupos vulnerables, desarrollar la comunicación educativa y crear productos para incrementar conocimientos, actitudes y prácticas; salud individual, familiar y colectiva; cultura en salud, autocuidado y autorresponsabilidad". Recuperado de: https://instituciones.sld.cu/upp/quienes-somos/
} 
Tabla 1. Ficha de análisis de contenido.

\begin{tabular}{|l|l|}
\hline \multicolumn{1}{|c|}{ Variables } & \multicolumn{1}{c|}{ Categorías } \\
\hline $\begin{array}{l}\text { 1)Propaganda Directa sobre el tema } \\
\text { COVID-19 }\end{array}$ & $\begin{array}{l}\text { a) Menciones } \\
\text { b) Promociones } \\
\text { c) Mensajes cortos } \\
\text { d) Jingles }\end{array}$ \\
\hline $\begin{array}{l}\text { 2)Propaganda Indirecta sobre el tema } \\
\text { COVID-19 }\end{array}$ & $\begin{array}{l}\text { a) Voz de locutor/a } \\
\text { b) Especialistas de diversas esferas } \\
\text { sociales }\end{array}$ \\
\hline $\begin{array}{l}\text { 3)Empleo de los recursos expresivos } \\
\text { radiales }\end{array}$ & $\begin{array}{l}\text { a) Voz } \\
\text { b) Música } \\
\text { c) Sonido } \\
\text { d) Efectos } \\
\text { e) Silencio }\end{array}$ \\
\hline $\begin{array}{l}\text { 4) Coherencia del contenido sobre } \\
\text { COVID-19 }\end{array}$ & $\begin{array}{l}\text { a) En la propaganda directa } \\
\text { b) En la propaganda indirecta } \\
\text { c) Entre la propaganda directa e indirecta. }\end{array}$ \\
\hline
\end{tabular}

Fuente: elaboración propia.

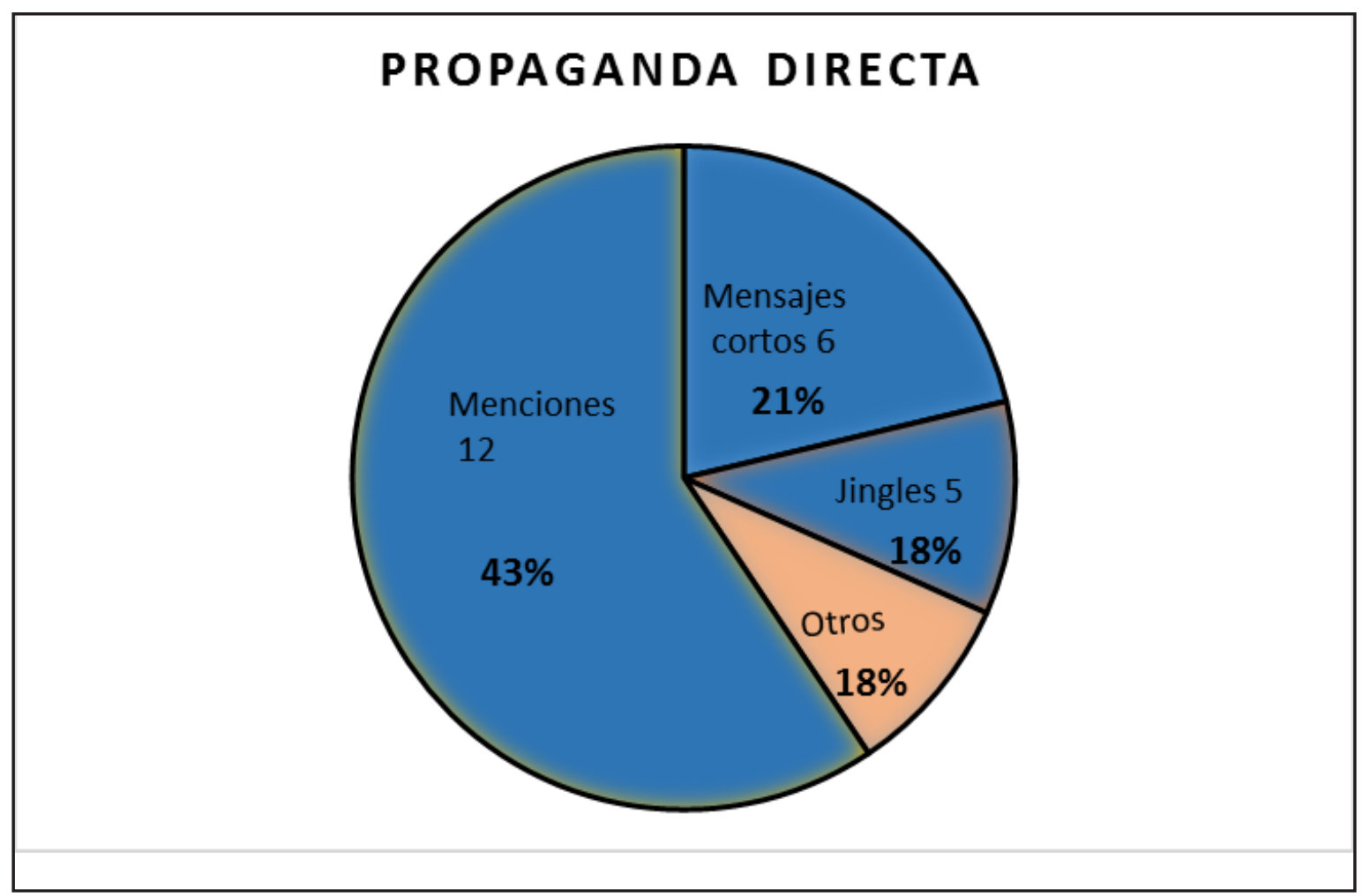

Figura 1. Porcentajes de la propaganda directa sobre el tema COVID-19. Fuente: elaboración propia 
El médico también indicó que es preciso organizar las visitas diarias que hace el equipo básico de salud, es decir, lo que se conoce como visita de terreno y enfatizar en los síntomas respiratorios, aunque aparentemente no esté relacionado con la COVID-19, dar atención a las enfermedades crónicas asociadas que pueda tener cada paciente, pues los casos más complicados y los fallecimientos, en su mayoría, manifiestan estos padecimientos. Destaca el aumento de las personas asintomáticas y la necesidad de cuidar a los adultos mayores porque no se sabe quién puede tener el virus.

Los recursos expresivos del medio fueron utilizados adecuadamente. Los locutores mantuvieron buena dicción y expresión oral en la conducción de los programas, en los cuales hubo presencia de voces femeninas y masculinas. Mientras, en la Propaganda Directa, el uso de las voces fue equitativo, pues tanto las femeninas como masculinas tuvieron 13 apariciones. En ocasiones, coincidían en una realización a dos voces.

Las letras de algunos de los números musicales estuvieron en relación con la temática de la COVID-19, tales como "Quiero verte otra vez" interpretada por Alexander Abreu y Habana de Primera o Arnaldo y su Talismán con "Vivir para vencer". La primera destaca la importancia de asumir una actitud positiva y responsable y la confianza en que se vencerá esta situación y, la segunda, en ocasión del 1 de mayo y la necesidad de celebrarlo desde las casas respetando al aislamiento para vencer la pandemia.

Es apreciable la prioridad que tiene el tratamiento de la temática sobre la COVID-19 tanto en el programa Juventud 2000 como en La Discoteca Popular. Desde la propaganda indirecta se percibe que, de forma reiterada, los locutores/ as de ambos programas, así como los invitados, mantienen el llamado a cumplir las medidas de prevención orientadas por las autoridades sanitarias. Lo hacen con expresiones persuasivas, sin autoritarismo y apelando a la actitud consciente y responsable de los ciudadanos.

Analizando el contenido de los relatos de ambos tipos de propaganda, se observa que existe coherencia hacia dentro de sus propios mensajes y entre la Indirecta y la Directa, pues se corresponde lo que se dice sobre la COVID-19 en las menciones, mensajes cortos y jingles y lo que expresan quienes participan en los programas en cada uno de los roles correspondientes.

Los números musicales transmitidos responden al perfil de ambos programas, incluso algunos seleccionados que se refieren a este tema de salud, llaman a actuar de forma disciplinada y cómo hacerlo para cortar la cadena de transmisión. Otros, no creados para el momento, también constituyen un apoyo en este sentido, como la canción "Valientes" del dúo Buena Fe, dedicada a los trabajadores cubanos internacionalistas, pero que en esta ocasión se relaciona con la relevancia del trabajo de todo el personal de la salud que lucha contra la pandemia y los que se encuentran en otros países del mundo con la ayuda solidaria para erradicarla. También "Contigo en la distancia", de César Portillo de la Luz, cuya frase es utilizada por la locutora de Juventud 2000 para insistir en la necesidad de mantener el aislamiento y quererse desde lejos para evitar el contagio.

En La Discoteca Popular, destaca el slogan "Quédate en casa que la radio te acompaña", el cual se reitera en la propaganda indirecta, así como en la Directa, por ejemplo, en los mensajes cortos, donde los artistas que emiten su mensaje concluyen expresando dicho slogan.

En la construcción de los relatos se emplean adecuadamente los recursos expresivos de radio, pues los textos son comprensibles, con términos claros y redactados de modo que se comprenden en la primera escucha. Es importante destacar que las voces de los locutores/as de estos programas habituales no son las oficiales, porque los profesionales que asumen estos roles exceden de 60 años y se encuentran en casa cumpliendo con el aislamiento orientado.

Las voces utilizadas, tanto femeninas como masculinas, son balanceadas. Sonidos y efectos están bien empleados, con adecuada edición. La música no compite con las voces y se utiliza la apropiada en cada realización, a pesar de no haberse creado, especialmente, para cada una de las formas de Propaganda Directa.

Situación similar ocurre durante la transmisión de ambos programas musicales, pues se destaca la calidad de quienes intervienen en su realización, como locutores/ as y especialistas. Entre estos últimos, es relevante el Doctor que interviene en la sección sobre la COVID-19 en Juventud 2000, que demuestra conocimientos sólidos y buen desenvolvimiento en el medio, cuya intervención no contradice lo escuchado en otros espacios del programa y en la Propaganda Directa transmitida.

\section{Conclusiones}

La actual pandemia "ha trastornado nuestra normalidad", sus afectaciones en todas las esferas sociales se sienten y se prevé largo período de recuperación, cuando ello sea posible. Su enfrentamiento debe ser multisectorial, lo que incluye a la comunicación.

La participación ciudadana responsable, consciente y disciplinada es muy importante para superar esta situación desfavorable de salud. Mantener informados a los ciudadanos y compulsarlos a su responsabilidad, 
debe ser uno de los objetivos de la comunicación en cada contexto que así lo exige.

En el carácter multisectorial de la lucha para superar esta crisis sanitaria, se tiene como fundamento la definición de salud de la Organización Mundial de la Salud (OMS) que la considera como "un estado de completo bienestar físico, mental y social, y no solamente la ausencia de afecciones o enfermedades" (OMS, 2006, p.1). Esta concepción está presente tanto en la incorporación de las diversas instituciones, organismos y organizaciones que contribuyen a esta lucha en el contexto cubano como en el contenido de los mensajes de comunicación.

La radio como medio de comunicación, crea mensajes que hacen referencia no solo a aspectos propiamente médicos, sino que abarcan otras aristas sociales para coadyuvar al éxito en esta lucha por la salud, de ahí que las comunicaciones sobre el Primero de Mayo se relacionan con la importancia de hacerlo desde el aislamiento y llamar a convertir a las casas en las plazas donde cada año el pueblo trabajador desfila o la relación entre la necesidad de estar en casa; pero velar por las medidas de ahorro de electricidad.

La comunicación desarrollada por la emisora Radio Progreso, específicamente en los programas Juventud 2000 y La Discoteca Popular, demuestra la prioridad que concede al tema sobre la COVID-19. A pesar de ser este el tema de prioridad de la comunicación en el país, las transmisiones de los contenidos en los programas analizados no saturan la escucha, pues se planifican adecuadamente, relacionándolos con las intervenciones de especialistas, algunos de los números musicales que se transmiten y su correspondencia con las menciones, mensajes cortos y jingles que se ponen al aire.

La comunicación de esta emisora es coherente con el Artículo 46 de la Constitución sobre el derecho de todas las personas a la salud, junto al derecho a la vida, la integridad física y moral, la libertad, la justicia, entre otros.

En ambos programas musicales está presente el slogan Quédate en casa, el cual es acompañado en La Discoteca Popular con la frase, La radio te acompaña, de este modo refuerza un rasgo que caracteriza a este medio desde su surgimiento que es servir de compañía a sus oyentes en cualquier lugar y circunstancia en que se encuentren.

Las emisiones del programa Juventud 2000 y La Discoteca Popular aquí analizadas, muestran cómo también en la programación musical es viable el tratamiento de cualquier tema, siempre que se conciba una comunicación bien pensada, planificada y sus relatos encierren contenidos expresados de forma clara, simple (no de simplista, sino comprensible) y concisa, máxime en la radio donde la forma de construir su narrativa debe ser comprendida por un solo sentido: el oído.

\section{Referencias bibliográficas}

Constitución de la República de Cuba. (2019). La Habana, Cuba: Editora Política.

Fonseca, B., y Saladrigas, H. (2019). Radioarte desde el concepto industria cultural. Revista Eletrônica Internacional de Economia Política da Informação, da Comunicação e da Cultura, 21(3), 101-118.

Kaplún, M. (2005). Producción de programas de radio. La Habana, Cuba: Pablo de la Torriente Brau.

Muñiz, M. (1990). Publicidad. Mito y Realidad en el Socialismo. La Habana, Cuba: Pablo de la Torriente.

Organización Mundial de la Salud. Documentos básicos, suplemento de la 45 edición. Recuperado de: https://www.who.int/governance/eb/ who_constitution_sp.pdf

Piñuel Raigada, J. L., y Gaitán Moya, J. A. (1995). Metodología general, conocimiento científico e investigación en la comunidad social [CD-ROM]. Madrid, España: Síntesis.

PROSALUD. (2020). Quienes somos. Recuperado de https://instituciones.sld.cu/upp/quienes-somos/

Ramonet, I. (2016). El imperio de la vigilancia. La Habana, Cuba: José Martí. 